\title{
Garroteamento da artéria coronária na revascularização do miocárdio. Relação entre o grau de aterosclerose e a lesão vascular: estudo experimental
}

Luís Roberto GEROLA*, Luis Antônio Ribeiro de MOURA*, Ênio BUFFOLO*, Luis Eduardo Villaça LEÃO*, Henrique Caivano SOARES*, Costabile GALLUCCI*

GEROLA, L. R.; MOURA, A. R.; BUfFOLO, E.; LEÃO, L. E. V.; SOARES, H. C.; GALLUCCI, C. - Garroteamento da artéria coronária na revascularização do miocárdio. Relação entre o grau de aterosclerose e a lesão vascular: estudo experimental. Rev. Bras. Cir. Cardiovasc., 2(1):64-69, 1987

RESUMO: As anastomoses safena ou mamária-coronária, sendo suturas realizadas em vasos de fino calibre, necessitam de condiçōes ideais para sua realização. Mesmo em circulação extracorpórea e pinçamento aórtico, pode persistir sangramento pela arteriotomia coronária, obrigando o cirurgiâo a realizar algumas manobras, entre elas os garroteamentos proximal e distal, para conseguir um campo exangue. Mais recentemente, face à possibilidade de se executar a revascularização miocárdica sem circulação extracorpórea, tornou-se fundamental dispor-se de um método que possibilite a oclusão temporária da artéria coronária para a realização das anastomoses. Com esta preocupação, foi realizado um estudo experimental, procurando avaliar seis métodos de hemostasia temporária, aplicados em artérias com graus variáveis de aterosclerose. O modelo experimental utilizado foi a artéria coronária direita, em cadáver. O estudo histológico, aplicando as colorações de hematoxilina-ecosina, Weigert e hematoxilina fosfotúngstica, permitiu a determinaçăo objetiva da intensidade da aterosclerose na artéria coronária e os padrôes e graus de lesôes causados à parede arterial pelos métodos utilizados para sua oclusão temporária. Nessa amostra, os resultados sugerem uma tendência de relacionamento direto entre a gravidade da lesão arterial induzida pelo garroteamento e a severidade da aterosclerose coronária, independente do tipo de dispositivo utilizado para a interrupção do fluxo coronário.

DESCRITORES: garroteamento coronário: lesāo vascular; aterosclerose coronária: miocárdio, revascularizaçāo.

\section{INTRODUCÃO}

A cirurgia de revascularização miocárdica consagrou-se como procedimento terapêutico, melhorando a qualidade de vida e, muitas vezes, aumentando a sobrevida dos pacientes portadores de aterosclerose coronária. A cirurgia coronária é um pro- cedimento bem padronizado, sendo executado com algumas variaçōes técnicas.

Geralmente, realizada sob pinçamento aórtico durante a circulação extracorpórea e com drenagem das câmaras esquerdas, muitas vezes persiste algum grau de sangramento pela arteriotomia

\footnotetext{
Trabalho realizado na Escola Paulista de Medicina. Săo Paulo, SP, Brasil

- Da Escola Paulista de Medicina.

Entregue para publicaçăo em 8 de abril de 1987.

Endereço para separatas: Luis Roberto Gerola. Rua Borges Lagoa, 783 5: andar. 04083 São Paulo, SP, Brasil.
} 
GEROLA, L. R.; MOURA, L. A. R.; BUfFOLO, E.; LEÃO, L. E. V.: SOARES, H. C.; GALLUCCI, C. - Garroteamento da artéria coronária na revascularizaçāo do miocárdio. Relação entre o grau de aterosclerose e a lesão vascular: estudo experimental. Rev. Bras. Cir. Cardiovasc., 2(1):64-69, 1987

coronária, necessitando alguma manobra hemostática; quando realizada sem o pinçamento aórtico, como preconizado por alguns autores ${ }^{3,6,8}$, sempre necessitará de manobras hemostáticas, para permitir a devida anastomose vascular com o campo operatório exangue.

Além disso, BUFFOLO et alii ${ }^{1}$ demonstraram, recentemente, a possibilidade do procedimento ser realizado sem a utilização do circuito de circulação extracorpórea. Esta técnica, usada freqüentemente em nosso Serviço, exige, para sua realização, o garroteamento das artérias coronárias.

Com o intuito de avaliar o grau de lesão do vaso coronário submetido ao garroteamento e tentar relacionar o grau da lesão com o grau da aterosclerose, realizamos um estudo, tendo, como modelo experimental, a artéria coronária direita em cadáver. Esta artéria foi escolhida pela freqüência com que é abordada e pelo seu padrão peculiar de aterosclerose, distribuído em todo o seu trajeto.

\section{MATERIAL E MÉTODO}

Foram utilizados 20 corações de cadáveres de ambos os sexos, cujas idades variaram de 40 a 60 anos, obtidos no Serviço de Verificação de Óbitos da Escola Paulista de Medicina, sendo que o tempo decorrido entre o óbito e a coleta do material não foi superior a 24 horas.

Foram usados seis métodos de hemostasia temporária, sob a forma de garroteamentos da artéria coronária direita aplicados após a margem direita do coração, sendo utilizados garrotes de plástico (equipo de soro) e garrotes de silicone (segmentos da sonda de Duboff) (Figura 1).

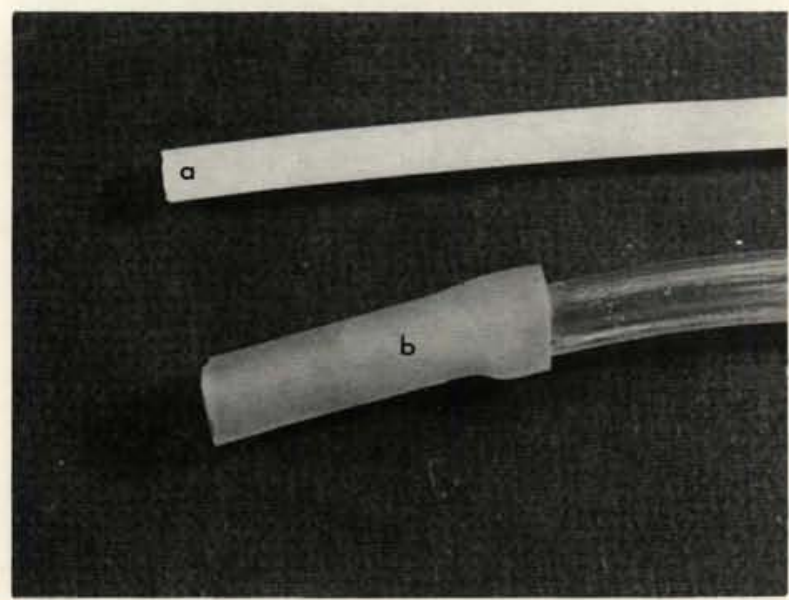

Fig. 1 - llustração das sondas utilizadas para o garroteamento coronário. Sonda de Duboff (A) e equipo plástico comum (B).
Os métodos usados foram: 1) garrote de equipo plástico comum com fio prolene $5-0$ sem coxim gorduroso periarterial; 2) pinça tipo Potts; 3 ) garrote de silicone com fio prolene 5-0 com coxim gorduroso periarterial; 4) garrote de silicone com fio Ethibond 2-0 com coxim gorduroso periarterial; 5) dupla laçada com fio prolene 5-0 com coxim gorduroso periarterial; 6) borracha de silicone flexível (vessel loop).

A aplicaçāo desses garrotes pode ser feita mantendo-se a gordura periarterial, obtendo-se, assim, um coxim gorduroso, ou com dissecção prévia, retirando-se a gordura periarterial (Figuras 2 e 3 ).

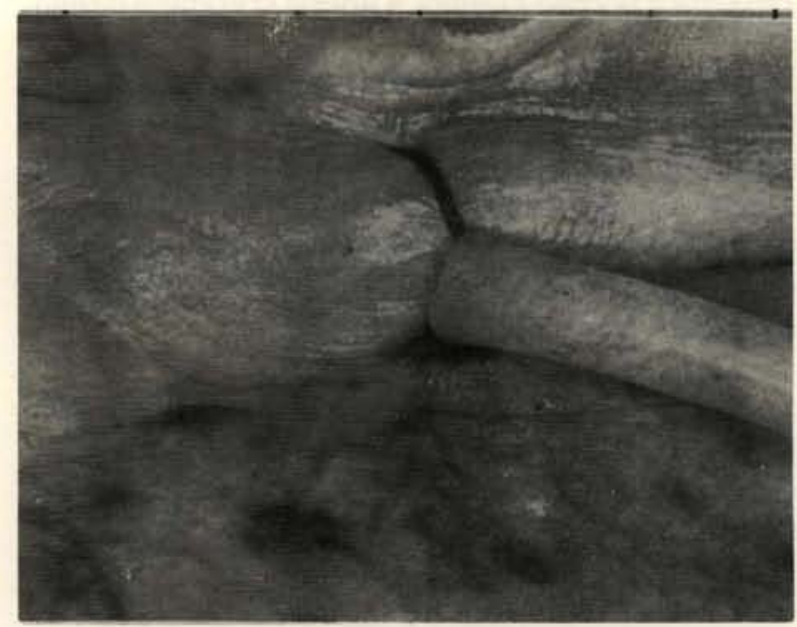

Fig. 2 - A ilustração representa a aplicação de garrote na artéria coronária com a gordura periarterial preservada.

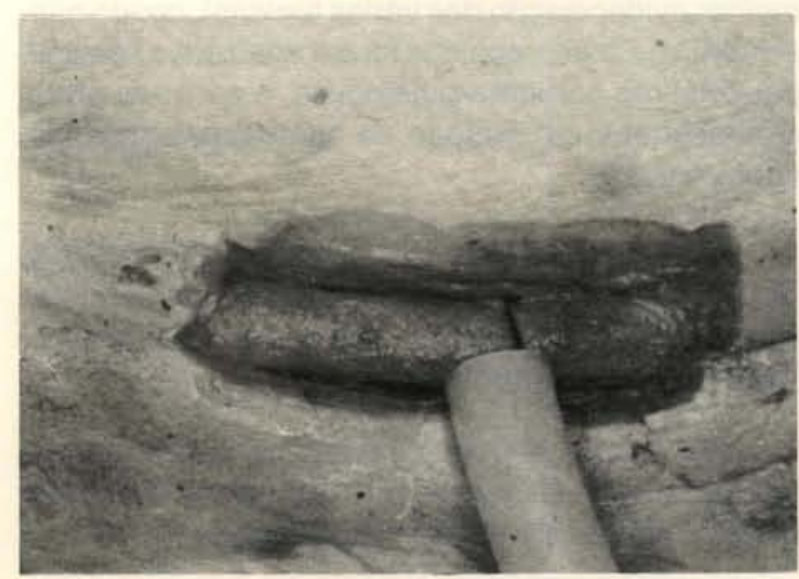

Fig. 3 - Representação de um garroteamento sobre a artéria coronária direita após a dissecção da gordura periarterial.

Com uma agulha curva, contorna-se a artéria coronária direita, penetrando no sulco atrioventricular e "laçando" a artéria. Com o auxílio de um passante, o fio é introduzido através do garrote, 
GEROLA, L. R.; MOURA, L. A. R.; BUFFOLO, E.; LEĀO, L. E. V.; SOARES, H. C.; GALLUCCI, C. - Garroteamento da arteria coronária na revascularização do miocárdio. Relação entre o grau de aterosclerose e a lesão vascular: estudo experimental. Rev. Bras. Cir. Cardiovasc., 2(1):64-69, 1987.

que é tracionado e a tensão é mantida prendendo-se o fio do garrote por meio de uma pinça tipo "mosquito".

$\mathrm{Na}$ dupla laçada, contorna-se a artéria coronária direita duas vezes, à semelhança de um chuleio simples, porém sem avançar o segundo ponto; após isto, aplica-se o garrote.

A utilização do vessel loop (borracha filiforme de silicone flexível) exige dissecção da região posterior à artéria e, com o auxílio de uma pinça tipo "mixter", o vessel loop é apreendido e contorna a artéria. Feito isto, tracionamos o suficiente para obter a oclusão do lúmen e é pinçada a borracha próxima à porção anterior à artéria coronária direita.

Em cada coração, foram aplicados dois desses métodos, na forma de garroteamento proximal e distal, por um período de 15 a 20 minutos, totalizando 40 garroteamentos. Com o auxílio de uma seringa com água, introduzida no óstio da artéria coronária direita, foi feita uma injeção para avaliar a eficácia dos garroteamentos.

Após o garroteamento, os segmentos da artéria coronária direita foram retirados, abertos longitudinalmente, fixados em líqüido de Bouin e enviados ao Serviço de Anatomia Patológica da Escola Paulista de Medicina.

Foram realizadas colorações pela hematoxilina-eosina, método de Weigert (para fibras elásticas) e hematoxilina fosfotúngstica de Mallory (para melhor evidenciação das várias túnicas), sendo que as análises microscópicas foram realizadas sempre pelo mesmo anátomo-patologista, o qual não tinha conhecimento do método de hemostasia aplicado sobre o vaso.

Durante a análise microscópica, procurou-se definir, inicialmente, o grau de aterosclerose do vaso, sendo classificado em três níveis de aterosclerose: leve, moderado e grave:

Aterosclerose leve: Espessamento discreto da intima, não ultrapassando, em espessura, o equivalente à metade da túnica média (Figura 4).

Aterosclerose moderada: Quando o espessamento da íntima equivale a cerca de metade da túnica média, além da presença de poucas placas com calcificação distrófica (Figura 5).

Aterosclerose grave: Presença de numerosas placas de ateroma, calcificações freqüentes e ulceraçōes (Figura 6).
As lesões encontradas foram, também, classificadas pelo anátomo-patologista, segundo ordem crescente de gravidade, em leve, moderada e grave, assim caracterizadas:

Lesão leve: Retificação da limitante elástica e discreto esmagamento da túnica média (Figura 7).

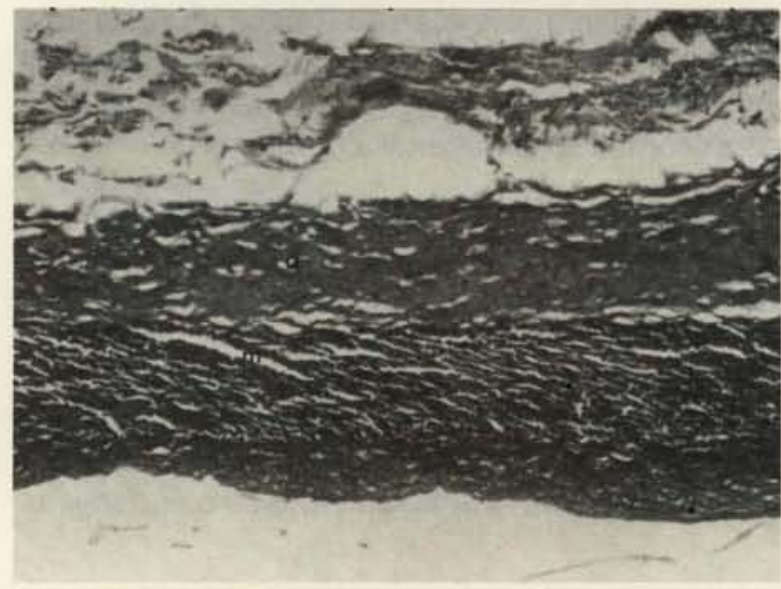

Fig. 4-Fotomicrografia de artéria coronária direita com aterosclerose leve. Observar discreto espessamento da túnica intima (i) não ultrapassando metade da túnica média. Coloraçăo de hematoxilina fosfotúngstica

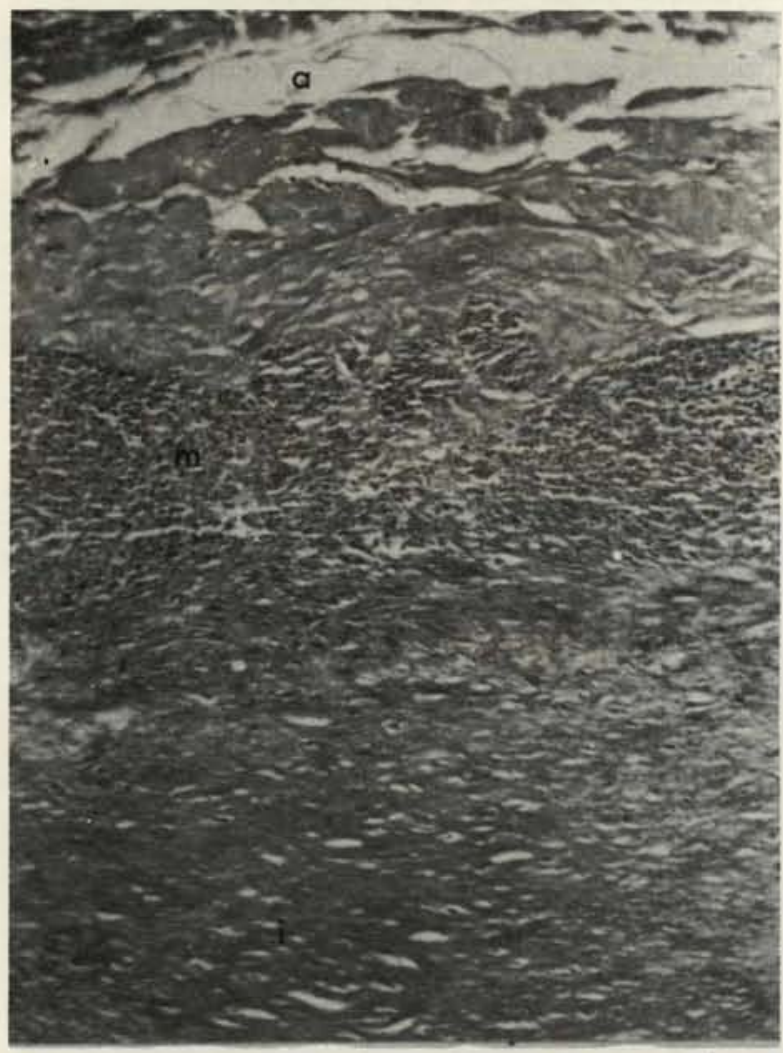

Fig. 5 - Fotomicrografia de artéria coronária direita com aterosclerose moderada. Grande espessamento da intima (i) ultrapassando o equivalente à metade da túnica média. Coloração de hematoxilina-eosina (HE). 
GEROLA, L. R.; MOURA, L. A. R.; BUfFOLO, E.; LEẢO, L. E. V.; SOARES, H. C.; GALLUCCI. C. - Garroteamento da artéria coronária na revascularização do miocárdio. Relação entre o grau de aterosclerose e a lesão vascular: estudo experimental. Rev. Bras. Cir. Cardiovasc., 2(1):64-69, 1987.

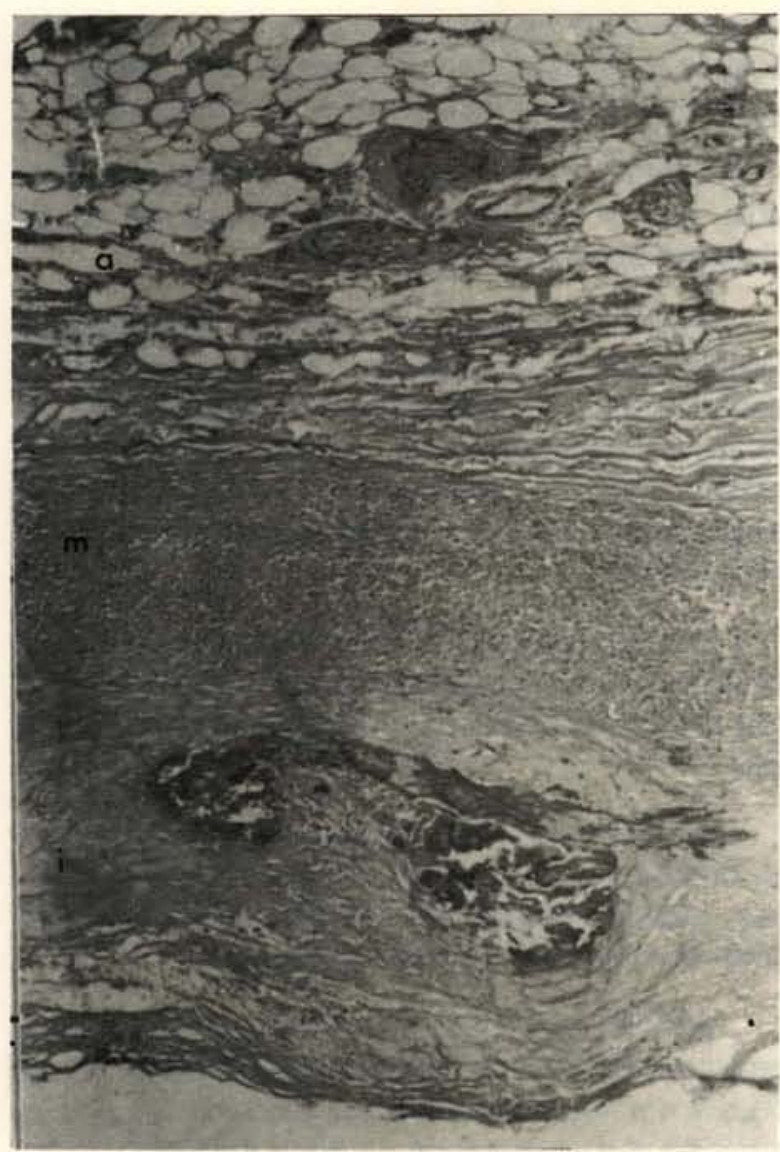

Fig. 6- Fotomicrografia da artéria coronária direita com aterosclerose grave. Grande espessamento intimal com placas de ateroma e ulceração. Coloração de hematoxilina-eosina (HE).

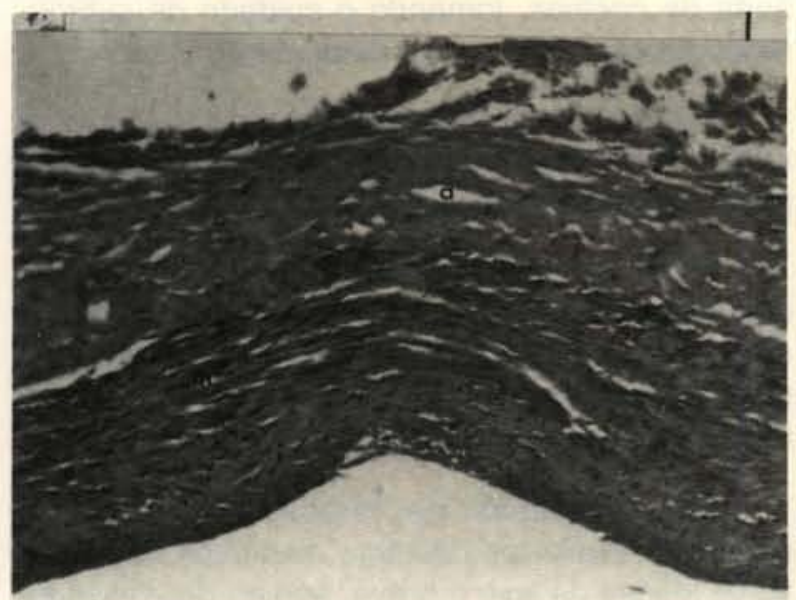

Fig. 7 - Fotomicrografia de artéria coronária direita com lesâo vascular de grau leve. Discreto esmagamento da túnica média. Coloração de hematoxilina fosfotúngstica.

Lesão moderada: Esmagamento da túnica média com perda da arquitetura e, eventualmente, de qualquer uma das membranas elásticas (Figura 8).

Lesãograve: Rotura total da média e das membranas elásticas (Figura 9).

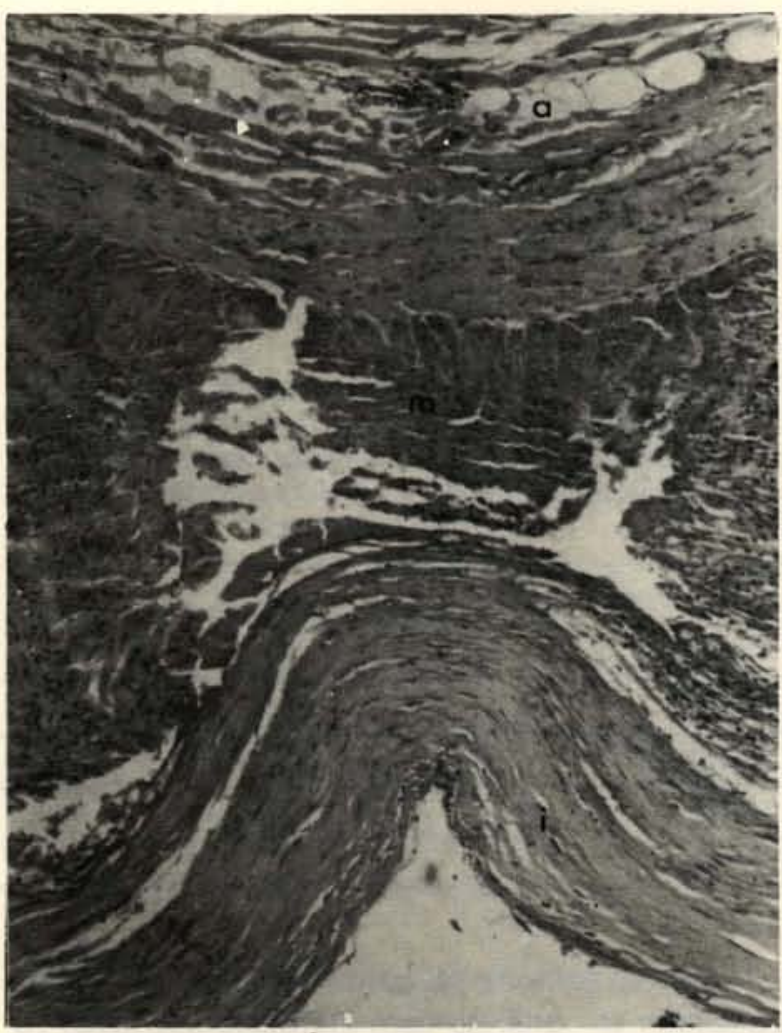

Fig. 8 - Fotomicrografia de artéria coronária direita com lesâo de grau moderado. Esmagamento da túnica média com perda de sua arquitetura e rotura de uma das membranas elásticas. Coloraçăo de hematoxilina-eosina

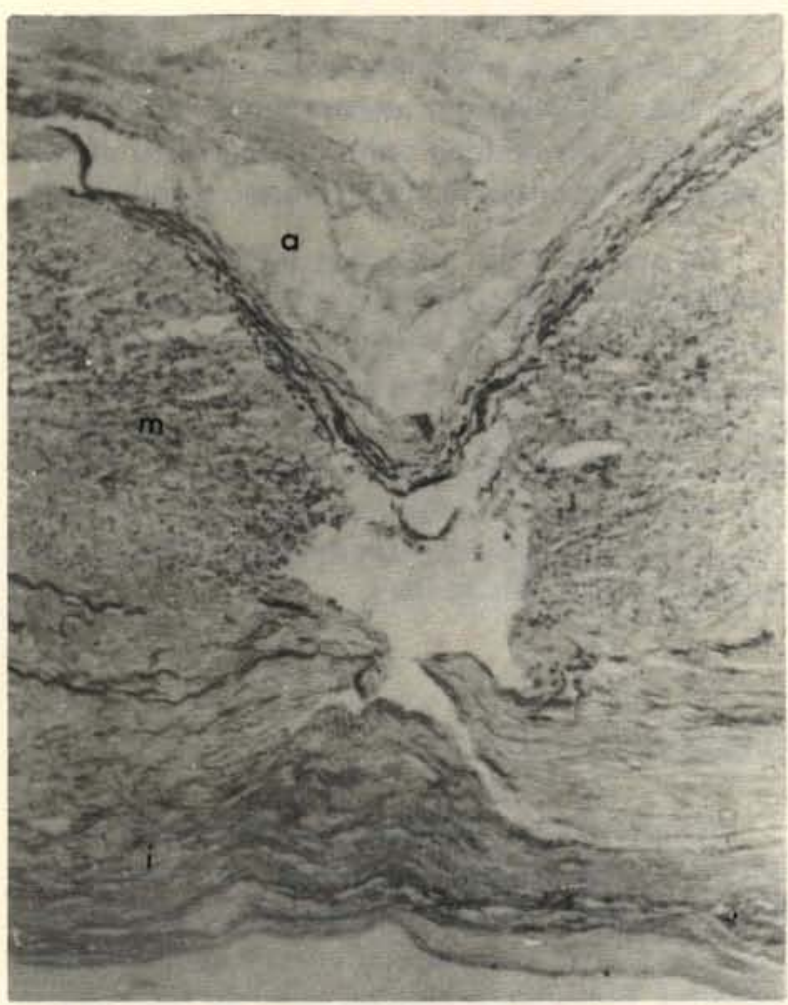

Fig. 9 - Fotomicrografia de artéria coronária direita com lesao grave. Rotura total da túnica média e secção das membranas elásticas. Coloração de Weigert. 


\section{RESULTADOS}

Os resultados obtidos estão representados na Tabela 1, onde é relacionado o grau de aterosclerose do vaso com o grau de lesão provocada por todos os métodos de hemostasia temporária.

\section{TABELA 1}

RELAÇAOO ENTRE LESĀO VASCULAREATEROSCLEROSE, INCLUINDO TODOS OS METTODOS DE HEMOSTASIA TEMPORARIA. OBSERVA-SE UMA TENDENNCIA DE RELACIONAMENTOENTREA LESAO VASCULAREO GRAU DE ATEROSCLEROSE

\begin{tabular}{|c|c|c|c|c|}
\hline Lesâa & LEVE & MODERADO & GRAVE & TOTAL \\
\hline LEVE & 8 & 6 & 3 & 17 \\
\hline MODERADO & 6 & 4 & 3 & 13 \\
\hline GRAVE & 0 & 4 & 6 & 10 \\
\hline TOTAL & 14 & 14 & 12 & 40 \\
\hline
\end{tabular}

De um total de 40 garroteamentos, 17 foram aplicados em artérias com aterosclerose leve, 13 em artérias com aterosclerose moderada e 10 em artérias com aterosclerose grave. Nas artérias com aterosclerose leve, $82 \%$ das lesões provocadas permaneceram entre leve ou moderada. Nas artérias com aterosclerose moderada, $76 \%$ das lesões provocadas permaneceram entre leve ou moderada. Nas artérias com aterosclerose grave, $60 \%$ das lesões provocadas eram graves, $40 \%$ das lesões eram moderadas, não havendo lesões leves.

De um total de 14 lesões leves provocadas, $100 \%$ delas foram em vaso com aterosclerose leve ou moderada. Das 14 lesōes moderadas provocadas, $71 \%$ foram em vasos com aterosclerose leve ou moderada, e $29 \%$ em vasos com aterosclerose grave. Das 12 lesōes graves provocadas, $50 \%$ foram em vasos com aterosclerose grave, $25 \% \mathrm{em}$ vasos com aterosclerose moderada e $25 \%$ em vasos com aterosclerose leve.

\section{DISCUSSĀO}

A hemostasia temporária, por definição, é aquela realizada para interromper, de forma transitória, a corrente sangüínea, mediante compressão vascular suave e delicada, com o mínimo de traumatismo. Portanto, o método ideal de hemostasia temporária é aquele que menor lesāo provocar ao vaso. Com o restabelecimento do fluxo sangüíneo, a presença de lesões vasculares favorece a trombose e pode comprometer per se a irrigação da área tratada, alterando o resultado da operação.

O estudo dos métodos de hemostasia temporária vem sendo desenvolvido a partir de vários modelos experimentais.

$\mathrm{EGDAHL}^{2}$ avaliou, inicialmente, as alteraçōes macroscópicas provodadas pelo pinçamento da aorta de cães. Posteriormente, HENSON \& ROB ${ }^{4}$ fizeram um estudo in vivo, durante cirurgias abdominais, testando várias pinças vasculares sobre a artéria gastroepiplóica direita, agora com análise microscópica.

HICKMAN \& MARTENSEN ${ }^{5}$, em seus estudos, avaliaram três importantes características das pinças vasculares: oclusão, preensão e o grau de lesão.

Nesses trabalhos, observa-se que os autores se preocupavam, fundamentalmente, com as pinças, fazendo poucas referências sobre as condições dos vasos onde estas atuavam.

WYLIE ${ }^{9}$, em 1954, já enunciava: "A aplicação de pinças vasculares hemostáticas sobre aorta que possui doença aterosclerótica provoca resultados que não sāo observados na aorta normal".

MASUOKA et alii ${ }^{7}$, para avaliar diversas pinças vasculares, usaram, como modelo experimental, aorta de coelhos, tomando o cuidado de submetê-los previamente a uma dieta aterogênica.

Neste material, observou-se uma tendência de relacionamento entre o grau de lesão provocada pelos garroteamentos e o grau de aterosclerose.

Assim, pode-se observar, na Tabela 1, que, quando a aterosclerose é classificada em leve e moderada, predominam lesōes leves e moderadas; no entanto, quando a aterosclerose é grave, predominam lesōes moderadas e graves.

Alguns métodos de hemostasia temporária mostram resultados bastante dispersos, apresentados lesões de leve a grave, apesar das mesmas condiçōes do vaso garroteado.

Entende-se que os garroteamentos são métodos realizados de modo empírico e a tensão dada aos garrotes varia de caso para caso, assim como são variáveis de operação para operação, ou mesmo de uma coronária para outra.

Acreditamos que este assunto mereça maior atenção e preocupação. É nossa intenção prosse- 
guir este estudo in vivo, em animais previamente submetidos a dieta aterogênica, propiciando, assim, o restabelecimento do fluxo sangüineo após o pinçamento, o que pode modificar o padrão das lesōes encontradas, "mimetizando" o melhor possível as condiçōes operatórias; poderemos, também, ampliar o estudo para outras coronárias, onde o padrão de aterosclerose pode ser diferente.

\begin{abstract}
RBCCV
GEROLA, L. R.; MOURA, L. A. R.; BUfFOLO, E.; LEÃO, L. E. V.; SOARES, H. C.; GALLUCCI, C. Occlusion of coronary artery in myocardial revascularization. Relationship between the degree of atherosclerosis and vascular injury: an experimental study. Rev. Bras. Cir. Cardiovasc.: 2(1):64-69, 1987

ABSTRACT: Coronary artery surgery, specially when performed without cardiopulmonary bypass, needs an "atraumatic" method that allows temporary coronary occlusion with minimal injury to the vessel wall. An experimental study was performed using the right coronary artery of cadaver hearts in order to evaluate the methods of "atraumatic" clamping of arteries with variable degrees of atherosclerosis. The vessels were evaluated histologically both for intensity of the atherosclerotic disease and intensity of injury to the coronary artery wall. Results suggest a relationship between severity of coronary artery disease and degree of injury to the artery wall.
\end{abstract} ization.

DESCRIPTORS: coronary occlusion; vascular injury; coronary atherosclerosis; myocardial revascular-

\section{REFERÊNCIAS BIBLIOGRÁFICAS}

1 BUfFOLO, E.; ANDRADE, J. C. S.; SUCCI, J. E.; LEÃO, L. E. V.; GALLUCCI, C. - Directal myocardial revascularization without cardiopulmonary bypass. Thorac. Cardiovasc. Surg., 33 (1): 26-29, 1985.

2. EGDAHL, R. H. - Simple clamp arterial anastomosis. Am. J. Surg., 91 (1): 113-114, 1956.

3. GREEN, G. E. - Internal mammary-coronary artery anastomosis for myocardial ischemia. In: SABISTON Jr., D. C. \& SPENCER, F. C. - Gibbon's surgery of the chest, 3. ed., Philadelphia, WB Saunders Co., 1976. p. 1378-1384.

4. HENSON, G. F. \& ROB, C. G. - A comparative study of the effects of different arterial clamps on the vessel wall. Br. J. Surg., 43 (182): 561-564, 1956.
5. HICKMAN, G. A. \& MORTENSEN, J. D. - A comparative evaluation of vascular clamps. J. Thorac. Cardiovasc. Surg., 44 (5): 561-569, 1962.

6. HUTCHINSON, J. E.; GREEN, G. E.; MEKHJIAN, H. A.; KEMP, H. G. - Coronary bypass grafting in 376 consecutive patients, with three operative deaths. J. Thorac. Cardiovasc. Surg., 67 (1): 7-15, 1974.

7. MASUOKA, S.; YOKOYAMA, A.; SHIMONURA, T.; KUROMOTO, N.; KAWAI, S. - Comparative evaluation of vascular clamps on the arteriosclerotic aortic walls. J. Vasc. Surg., 14 (4): 243-254, 1980.

8. SPENCER, F. C. - Bypass grafting for oclusive disease of the coronary arteries. In: SABISTON Jr., D. C. \& SPENCER, F. C. - Gibbon's surgery of the chest. 3. ed., Philadelphia, WB Saunders Co., 1976. p. 1364-1375.

9. WYLIE, E. J. - A new aorta clamp. Surgery, 36 (1): 781-789, 1954 